\title{
POSTER CONTRIBUTIONS
}

The abstracts of the poster contributions presented at I.A.U. Symposium $n^{\circ} 191$ have been published within a separate book distributed to the participants during the Conference. An electronic version of these abstracts can be consulted at:

http://www.dstu.univ-montp2.fr/GRAAL/agb98/BOOK/prog/program.html These abstracts are also archived in the Astrophysics Data System (ADS) of the N.A.S.A.

\section{Basic Facts, Structure, Evolution, Nucleosynthesis}

Abia C., de Laverny P., Pavlenko Y.

New and accurate Li abundance determinations in super Li-rich AGB stars

Amari S., Nittler L.R., Zinner E., Lewis R.S.

Isotopic analyses of presolar SiC grains of type $Y$

Cioni M.R., Habing H.J., Loup C., Epchtein N.

DENIS: Color-Magnitude diagrams and Luminosity Function towards the Magellanic Clouds

Decin L., Cohen M., Eriksson K., Gustafsson B., Huygen E., Morris P., Plez B., Sauval J., Vandenbussche B., Waelkens C.

Modelling of ISO-SWS spectra of red giants

Domínguez I., Straniero O., Isern J.

$A G B$ stars as Astroparticle Laboratories

Driebe T., Blöcker T., Herwig F., Schönberner D.

Diffusive overshooting in hot bottom burning AGB models

Dufour E., Kahane C., Greaves J.C.

Modelling ${ }^{12} \mathrm{C} /{ }^{3} \mathrm{C}$ ratios

Gallino R., Busso M., Wasserburg G.J., Straniero O.

Short-lived Isotopes from a Close-by AGB Star Triggering the Protosolar Nebula

Girardi L.

Constraints to the $A G B$ evolution provided by $A G B$ and white dwarf stars in clusters, and integrated cluster colours

Herwig F., Schönberner D., Blöcker T.

Violation of the Core Mass - Luminosity Relation for AGB models which experience the third dredge-up 
Josselin E., Blommaert J.A.D.L., Groenewegen M.A.T., Omont A.

Identification and Characterization of Red and Infrared Supergiants

Klochkova V.G.

Chemical Composition of atmospheres of stellar IRAS-sources

Lugaro M., Gallino R., Arlandini C., Busso M., Straniero O.

Sr Isotopic Composition in AGB SiC Grains

Mazzitelli I., D'Antona F., Ventura P.

Lithium formation in massive AGB stars: new models

Ohnaka K., Tsuji T.

Elemental Abundance Analysis in Carbon Stars

Rauscher T., Thielemann F.-K.

Astrophysical reaction rates for nucleosynthesis in AGB stars

Ryde N., Eriksson E., Gustafsson B., Olofsson H., Plez B.

Modelling of molecular bands of oxygen-rich AGB stars

\section{Pulsation, Mass Loss, Cool Envelopes}

Andronov I.L.

Multiple periodicities in long-period variables

Balklavs A., Dzervitis U., Eglitis I.

Analysis of absolute magnitudes of carbon stars from HIPPARCOS parallaxes

Barthès D., Luri X.

Luminosity calibrations and kinematics of Galactic oxygen-rich LPVs: derivation of $P-L-C$ relations and modelling

Broc A., Lafon J.-P.J.

Non-equilibrium radiative hypersonic flows

Cami J., de Jong T., Tielens A.G.G.M., Justtanont K., Waters L.B.F.M., Yamamura I.

Gas-phase $\mathrm{CO}_{2}$ around $\mathrm{O}$-rich $\mathrm{AGB}$ stars

Carpenter K.G.

The Structure of the Outer Atmosphere and Wind of $\lambda$ Vel

Chinarova L.L.

Time series analysis of the long-term variability of the giant components in symbiotic variables 
Duari D., Cherchneff I., Willacy K.

Carbon-bearing species in the inner wind of oxygen-rich Miras

Gray M.D.

The Natural Saturated Beam Angles of Circumstellar SiO Masers

Helling Ch., Woitke P., Winters J.M., Sedlmayr E.

Influence of molecular opacities on the generation of cool stellar winds

Herpin F., Baudry A., Alcolea J., Cernicharo J.

On the high velocity SiO maser emission from late-type stars

Jiménez-Esteban F., García-Lario P., Manchado A., Engels D. Identification and monitoring program of $O H / I R$ stars

de Jong T., Yamamura I., Cami J., Onaka T., Waters L.B.F.M.

Discovery of the $\mathrm{SO}_{2} 7.3 \mu \mathrm{m}$ band in Oxygen-rich Miras

Jorissen A., Udry S.

Shock waves in Mira stars : the pseudo SB2 orbit of the CS star RZ Peg

Justtanont K., de Jong T., Tielens A.G.G.M., Cami J., Waters L.B.F.M., Yamamura I.

SWS Observations of Supergiants

Kudashkina L.S., Andronov I.L.

Atlas of the mean light curves of the Mira-type stars

Lebzelter T., Hinkle K.H., Hron J.

Atmospheric Kinematics of Long period Variables

Lewis B.M.

Mass-Loss and Gravitational Contraction

Linsky J.L., Harper G.M., Bennett P.D., Brown A., Valenti J.

A Critical Evaluation of Mass Loss Rates and Wind Properties of Evolved Latetype Stars

Marsakova V.I., Andronov I.L.

Correlations between the characteristics of the individual cycles of the Mira-type stars

Matsuura M., Yamamura I., Murakami H., Freund M.M.

The Observation of $\mathrm{H}_{2} \mathrm{O}$ in M-giants in the Near-and Mid-Infrared Region with the Infrared Telescope in Space (IRTS) 
Mattei J.A., Foster G.

Trend Analysis of Oxygen-Rich Long-Period Variables

McNamara D.H.

Period-Luminosity relations of Populations II Cepheids

Netzer N.

Radiative Transfer and Dynamics of Circumstellar Envelopes of Variable Red Giants

Perrin G., Coudé du Foresto V., Ridgway S.T., Mariotti J.-M., Ruilier C., Mennesson B., Traub W.A.

Direct Evidence of the Pulsation of the Photosphere of AGB Stars

Schultheis M., Aringer B., Höfner S., Jørgensen U.

TiO bands in Miras and Semi-regular Variables

van Belle G., The PTI Collaboration, Thompson R.R.

Evolved Star Sizes, Temperatures as Directly Measured with Interferometry

Wallerstein G., Barnbaum C.

U Equ: A Unique Variable with Strong TiO Emission

Windsteig W., Aringer B., Lebzelter T., Höfner S.

$4 \mu \mathrm{m}$ High Resolution Spectra of AGB stars

Wing R.F., Alvarez R., Plez B., Yuan Y.

Behavior of Mira Variables in Bandstrength-Color Diagrams. Comparison of Dynamic Models with Calibrated Spectral Scans

\section{Formation, Composition, and Processing of Dust}

Andersen A.C., Jørgensen U.G., Henning Th., Mutschke H.

Laboratory study of dust from AGB stars

Cau P., Cherchneff I.

The formation of aromatics and metal carbides in the inner wind of carbon stars

Chamaillard K., Lafon J.-P.J.

Effects of rough surfaces on optical properties of grains

Dirks U., Sedlmayr E.

The influence of temperature fluctuations on dust formation in cool stellar atmospheres 
Ferguson J.W., Alexander D.R., Johnson H.R., Allard F., Hauschildt P.H. The Formation of PAHs in Carbon Star Atmospheres

Jura M.

Scientific opportunities with SIRTF

Koike C., Suto H., Tuchiyama A., Shibai H., Tanabe T.

The spectra of pyroxenes in mid and far infrared

Le Bertre T., Winters J.M., Sedlmayr E.

The mass loss rates of $A G B$ stars from near-infrared photometry

Liberatore S., Lafon J.-P.J.

Modelling of dynamics in circumstellar dust shell

Lobel A., Doyle J.G., Bagnulo S.

Modelling the Spectral Energy Distribution of Carbon and Oxygen-rich stars from stellar model spectra

Lodders K., Fegley B. Jr.

The influence of metallicity on dust condensation temperatures in circumstellar envelopes of giant stars

Marengo M., Busso M., Silvestro G., Fazio G.

$A G B$ circumstellar envelope dust mineralogy from ground based mid-IR imaging and photometry

Molster F., Waters L.B.F.M., Jäger C., Henning Th.

The crystalline silicates of AFGL 4106

Patzer A.B.C., Chang Ch., John M., Sedlmayr E.

On the formation of inorganic clusters in oxygen-rich circumstellar envelopes of $A G B$ stars

Pompeia L., Lorenz-Martins S.

Dust envelope models and spectroscopic study of oxygen-rich stars: corundum and silicate grains

Ryde N., Eriksson K., Gustafsson B., Olofsson H., Wolstencroft R., Nyman L.- $\AA$. Mid and far-infrared spectra of the third brightest carbon star IRAS 15194-5115

Simis Y.

Two fluid hydrodynamics of dust driven $A G B$ winds

Speck A.K., Hofmeister A.M., Barlow M.J.

Resolution of the SiC problem: astronomical and meteoritic evidence reconciled 
Suto H., Koike C., Shibai H., Tuchiyama A., Mizutani K.

The optical constants of crystalline silicate particles in mid-and far-infrared

Voshchinnikov N.V.

Diagnostics of dust properties from polarimetric observations of late-type stars

Woitke P., Sedlmayr E.

Thermal Bifurcations in Circumstellar Envelopes of C-Stars

\section{Circumstellar Envelopes}

Chapman J.M., Rudnitskij G.M.

Shock Waves and Radio Continuum in Miras

Colomer F., Reid M.J., Menten K.M., Bujarrabal V.

The spatial and frequency structure of circumstellar water masers

Crosas M., Young K., Ivezic Z., Knapp G.R.

New Atomic Carbon Detections in post-AGB stars

Delgado D.G., Olofsson H., Schwarz H., Eriksson K., Gustafsson B.

Images in scattered light of detached circumstellar shells

Etoka S., Szymczak M., Le Squeren A.-M.

Variability of $\mathrm{OH}$ masers in Semi-regulars

Gérard E.

The kinematics and dynamics of $O H$ masers in circumstellar envelopes of $A G B$ stars

Hashimoto O., Izumiura $\mathrm{H}$.

Circumstellar dust envelopes of oxygen-rich AGB stars with intermittent mass loss

Irrgang P., Balega Y.Y., Gauger A., Osterbart R., Schniggenberg G., Weigelt G. Speckle masking imaging and radiative transfer modeling of the oxygen-rich dust shells of AFGL 2290 and CIT 3 (WX Psc)

Käufl H.U., Stecklum B., Richichi A., Richter S.

AGB-Star Diameters Measured in the mid-Infrared Using Lunar Occultations

Kerschbaum F., Olofsson H., Larsen F., Bergman P.

$\mathrm{mm} /$ submm-spectroscopy and -interferometry imaging of envelopes of O-rich Semiregular and Irregular Variables 
Larsen F., Olofsson H.

Circumstellar envelopes of carbon stars

de Laverny P., Mauron N., Lopez B.

Observations and models of $A G B$ dust envelopes illuminated by ambient galactic light

Masheder M.R.W., Richards A.M.S., van Langevelde H.J.

$V L B I$ Observation of $\mathrm{OH}$ stars

Nyman L.-Å., Olofsson H., Schwarz H., Sahai R.

The bipolar outflow from the M-giant HR 3126

Olofsson H., Bergman P., Lucas R., Eriksson K., Gustafsson B., Bieging J.H. Episodic mass loss of the carbon star TT Cyg

Sivagnanam P., David P.

Anomalies of $\mathrm{OH}$ maser profiles in circumstellar envelopes: Overshoot of $\mathrm{OH}$ $1667 \mathrm{MHz}$ masers

Szymczak M., Cohen R.J., Richards A.M.S.

Polarization structure of $\mathrm{OH}$ masers in circumstellar shells

van Langevelde H.J., Diamond P.J., Schilizzi R.T., Baudry A., Habing H.J.

$V L B I$ measurements of the parallax and proper motion of $U$ Herculis

Yates J., Sylvestor R.

Modelling the MIR and FIR radiation transport of $\mathrm{CO}$ and $\mathrm{H}_{2} \mathrm{O}$ in the circumstellar envelopes of late-type stars

\section{Non-spherical Mass Loss, Binarity, Post-AGB Evolution}

Buscher D.F., Haniff C.A., Oudmaijer R.D.

Asymmetric structures in the circumstellar envelopes of planetary nebula progenitor stars

Dayal A., Sahai R.

Optical/Infrared Imaging and 3-Dimensional Spatial and Kinematical Modeling of Proto-Planetary and Planetary Nebulae

Fong D., Meixner M., Sutton E., Bujarrabal V., Kelly D., Haas M., Barlow M., Glassgold A., Nguyen-Q-Rieu, Skinner C., Tielens A.G.G.M.

Observations of Atomic Gas in Post-AGB Envelopes

Frankowski A., Tylenda R.

The AGB phase in binaries 
Gledhill T., Chrysostomou A., Yates J., Efstathiou A., Ménard F., Chikami K. Imaging Polarimetry of Circumstellar Dust Envelopes: The Detached Shell around IRAS 19114+0002

Harrington J.P., Borkowski K.J., Blondin J.M.

HST Polarization Observations of the Young Post-AGB Object He 3-1475 and the Origin of Bipolar Jets

Hinkle K., Fekel F., Joyce R.

Orbits for $A G B$ binary systems

Hrivnak B.J., et al.

A Study of Variability in Post-AGB Stars

Hua C.T., Dopita M.A.

Detection of new emission structures around planetary nebulae

Hurley J., Pols O., Tout C.

$A G B$ stars in Binaries

Karakas A.I., Tout C.A., Lattanzio J.C.

The eccentricities of the barium stars

Meixner M., Ueta T., Dayal A., Bobrowsky M., Hrivnak B.J., Skinner C.J., Hora J., Deutsch L.K., Hoffman W.F., Fazio G.

The Morphology of Proto Planetary Nebulae Dustshells

Mikolajewska J., Ivison R.I., Omont A.

Mass loss from red giants in symbiotic binaries

North P.

Fundamental parameters of $B a$ dwarfs and $C H$ subgiants

Ortiz R., Castilho B.V.

Atmospheric parameters and abundance analysis of post-AGB candidates

Osterbart R., Balega Y.Y., Langer N., Men'shchikov A.B., Weigelt G.

75 mas Speckle Imaging and Radiative Transfer Modeling of the Red Rectangle

Pooley D.J., Albrow M.D., Pollard K.R., Cottrell P.L.

Spectroscopic monitoring of southern post-AGB stars

Rudnitskij G.M.

Effects of a Close Low-mass Companion on the Spectrum and Light Curve of a Mira-type Star 
Sahai R.

Towards a New Understanding of the Formation of Aspherical Planetary Nebulae from $A G B$ stars: The Impact of $H S T$

Soker N.

Axisymmetrical final glory of dying stars

Su K.Y.L., Kwok S., Volk K., Hrivnak B.J.

WFPC2/HST Imaging of the Bipolar Proto-Planetary Nebulae

Sweigart A.V.

Radiation-Pressure Ejection of Planetary Nebulae in Asymptotic-Giant-Branch Stars

Szczerba R., Jeske K., Shematovich V., Zalfresso-Jundzillo M., Volk K.

Hydrodynamical modelling of the post-AGB phase of stellar evolution: dust and molecular gas emission

Van Eck S., Jorissen A., Mayor M., Udry S., Burnet M.

Extrinsic and intrinsic $S$ stars in the Henize sample

Villaver E., Manchado A., García-Segura G., Guerrero M.

Hydrodynamical simulations of Multiple Shell Planetary Nebulae

Volk K., Kwok S., Hrivnak B.

ISO Observations of Proto-Planetary Nebulae and Carbon Stars

Začs L., Nissen P.E., Schuster W.J.

HD 196944: a carbon and s-process rich, very metal-poor star

\section{AGB Stars as a Population of Various Galaxies}

Blommaert J., Groenewegen M., Trams N., Cioni M.R., Habing H., Okumura K., van Loon J., Loup C., Waters R., Zijlstra A.A.

Evolution and mass loss of AGB stars in the Magellanic Clouds

Dopita M.A., Vassiliadis E., Wood P.R., Meatheringham S.J., Harrington J.P., Bohlin R.C., Ford H.C., Stecher T.P., Maran S.P.

HST observations of Magellanic Cloud Planetary Nebulae

Exter K., Barlow M., Clegg R., Walton N., Parker Q.

Chemistry of the Galactic Bulge

Jean C., Terzan A., Guibert J.

Characteristics and IRAS Identifications of 4000 Long Period Variables detected in a field towards the Galactic Bulge 
Jehin E., Magain P., Neuforge C., Noels A., Parmentier G., Thoul A.A. The impact of $A G B$ stars on the chemical evolution of globular clusters

Kholtygin A.F.

New CNO abundances: test for the Galaxy chemical evolution

Matsumoto S., Nakada Y., Glass I.S.

PANIC Survey of the Galactic Bulge - The Duration Time of High Mass Loss Phase

Ojha D.K., Omont A., Simon G., ISOGAL team

Mass-losing AGB stars in the ISOGAL survey

Pols O., Hurley J., Tout C.

An efficient evolution algorithm for population synthesis of $A G B$ stars 